\title{
Germinación de genotipos de pasto banderita (Bouteloua Curtipendula) bajo diferentes presiones osmóticas
}

\section{Germination of sideoats grama (Bouteloua Curtipendula) genotypes under different osmotic pressures}

\author{
Alan Álvarez-Holguín ${ }^{1}$, Carlos Raúl Morales-Nieto ${ }^{1 *}$, Raúl Corrales-Lerma ${ }^{1}$, Alicia Melgoza-Castillo ${ }^{1}$, \\ Gerardo Méndez-Zamora ${ }^{2}$ \\ ${ }^{1}$ Facultad de Zootecnia y Ecología. Universidad Autónoma de Chihuahua. Periferico Francisco R. Aldama, Km 1. CP. 31000, \\ Chihuahua, Chihuagua, México \\ ${ }^{2}$ Centro de Investigación y Desarrollo en Industrias Alimentarias. Facultad de Agronomía. Universidad Autónoma de Nuevo \\ León. Ex Hacienda El Canadá, CP. 66050, General Escobedo, Nuevo León, México \\ *Autor de correspondencia: cnieto@uach.mx
}

Nota científica recibido: 15 de septiembre de 2015, aceptado: 15 de mayo de 2016

RESUMEN. El objetivo fue evaluar la respuesta de germinación de siete genotipos de pasto banderita (Bouteloua curtipendula), sometidos a diferentes niveles de presión osmótica. Los genotipos utilizados fueron El Reno (Re), Niner (Ni), Vaughn (Va), Kansas-6113 (K-13), Kansas-6107 (K-07), ecotipo 689 (E-689) y ecotipo 592 (E-592). Como agente osmótico se utilizó manitol, los niveles de presión osmótica fueron 0.0, -0.7, -1.4 y -2.1 Megapascales. Las variables evaluadas fueron porcentaje de germinación (GE), índice de velocidad de germinación (Vge), longitud de radícula (LR) y plúmula (LP). Los genotipos nativos (E-592 y E-689) y Va presentaron mayor resistencia al estrés hídrico $(\mathrm{p}<0.05)$. El genotipo K-07 presentó menor GE y Vge, mientras que Ni no presentó germinación a -2.1 MPa. Los genotipos E-592, E-689 y Va pueden considerarse en programas de rehabilitación de pastizales. Sin embargo, por su velocidad de germinación se recomienda utilizarlo mezclado con Re o K-13.

Palabras clave: Ecotipo; estrés hídrico; manitol; megapascales; pasto nativo.

ABSTRACT.The objective was to evaluate the germination rate of sideoats gamma (Bouteloua curtipendula) genotypes under different osmotic pressure levels. The genotypes used were El Reno (Re), Niner (Ni), Vaughn (Va), Kansas-6113 (K-13), Kansas-6107 (K-07), ecotype 689 (E-689) and ecotype 592 (E-592). The osmotic agent employed was mannitol. The osmotic pressure levels were; $0.0,-0.7,-1.4$ and -2.1 Megapascals (MPa). The variables evaluated were germination percentage (GE), germination speed index (GSI), radicle length $(R L)$ and plumule length (PL). The native genotypes (E-592 and E-689) and VA showed the highest resistance to water stress. The genotype K-07 presented the lowest GE and GSI, while Ni did not germinate under $-2.1 \mathrm{MPa}$. Based on the results, E-592, E-689 and Va can be considered for their inclusion on grassland rehabilitation programs. However, due to their speed germination it is recommended to use them in mixture with Re or K-13.

Key words: Ecotype; mannitol; megapascals; native grass; water stress.

\section{INTRODUCCIÓN}

La germinación, emergencia y supervivencia de plántulas son etapas críticas durante el establecimiento de las plantas. En las zonas áridas y semiáridas el desecamiento de la superficie del suelo se presenta con mayor rapidez después de un evento de lluvia, lo cual reduce las tasas de germinación (Esqueda et al. 2005). Por otra parte, la presión osmótica indica la fuerza que ejerce una planta o semilla para absorber agua del suelo, por lo que durante una sequía las plantas tienen que ejercer mayor turgencia para disponer de agua (Aparecida y Zambello 2003). Sin embargo, algunas 
especies tienen mayor capacidad para ejercer presión osmótica, lo que es una ventaja durante el establecimiento en zonas de escaza precipitación (Silmara y Juliano 2004). Aunado a lo anterior, algunas plantas aumentan el crecimiento de sus raíces a mayor profundidad con el propósito de captar el agua menos ligada al suelo y mantener una presión osmótica mínima durante la sequía (Trachsel et al. 2010, Aasamaa y Sõeber 2011). En este sentido, la capacidad de germinación y emergencia que tiene una semilla es un aspecto fundamental para el establecimiento y supervivencia (Springer 2005). Para simular condiciones de sequía y salinidad en especies vegetales se han utilizado agentes osmóticos como manitol, polietilenglicol, sulfato de potasio o cloruro de sodio. La inhibición de la germinación con soluciones de polietilenglicol y manitol se atribuye a un efecto osmótico, mientras que las soluciones de sulfato de potasio y cloruro de sodio disminuyen el potencial osmótico por intoxicación iónica (Ruiz y Torenti 2012, Méndez et al. 2010).

La evaluación de materiales resistentes al estrés hídrico, es una alternativa para la selección de genotipos adecuados a zonas de escaza precipitación. Simulaciones de este tipo se han realizado en especies como maíz (Zea mays L.), frijolillo (Rhynchosia minima), pasto buffel (Pennisetum ciliare), Klein (Panicum coloratum) y otras especies nativas del norte de México como el pasto banderita (Bouteloua curtipendula) (Méndez et al. 2010, Biligetu et al. 2011, Ruiz y Torenti 2012). Por su contenido de nutrientes y su alta aceptación por el ganado, el pasto banderita es considerado como la segunda especie forrajera en importancia ganadera del norte de México (Morales-Nieto et al. 2006). Algunas variedades de este pasto son Diana, Premier, Butte, Haskell, Trailway, Killdeer, Pierre, Niner, Vaughn, y El Reno, las cuales han sido desarrolladas en diferentes partes de Estados Unidos y México. Estas variedades cuentan con aptitudes para establecerse en zonas áridas y semiáridas de México, y pueden incluirse en programas de resiembras o rehabilitación de agostaderos (Beltrán et al. 2010, Lloyd-Reilley y Smith 2012). Por lo anterior, el objetivo de la presente investigación fue evaluar la respuesta germinativa de genotipos de pasto banderita (Bouteloua curtipendula) en condiciones de estrés hídrico, bajo diferentes niveles de presión osmótica.

\section{MATERIALES Y MÉTODOS}

El presente trabajo se realizó en el laboratorio de Parámetros Ambientales de la Facultad de Zootecnia y Ecología de la Universidad Autónoma de Chihuahua. Las variedades comerciales de pasto banderita utilizadas fueron El Reno (Re), Niner (Ni), Vaughn (Va), Kansas 6113 (K-13) y Kansas 6107 (K-07), procedentes de USA. También se se incluyeron los ecotipos 689 (E-689) y 592 (E-592), los cuales fueron recolectados en diferentes municipios del estado de Chihuahua en el 2008 y establecidos en el Campo Experimental La Campana del INIFAP (Morales et al. 2009). E-689 se recolectó en el km 68.5 carretera el Sauz-Namiquipa en un pastizal amacollado; mientras que E-592 se colectó en el km 51 de la carretera de cuota Ojinaga-Chihuahua en un matorral inerme parvifolio. Para homogeneizar el término variedades y ecotipos se utilizó el término genotipo.

Como agente osmótico se utilizó manitol $\left(\mathrm{C}_{6} \mathrm{H}_{1} 4 \mathrm{O}_{6}\right)$ diluido en agua destilada con diferentes concentraciones. Los tratamientos utilizados para simular el nivel de presión osmótica de las semillas fueron: $0.0,-0.7,-1.4$ y -2.1 Megapascales (MPa). Las concentraciones del glúcido se calcularon de acuerdo a su peso molecular $\left(182.17 \mathrm{~g} \mathrm{~mol}^{-1}\right)$ con la ecuación propuesta por Vant'Hoff (Ruiz y Torenti 2012): $\Psi \pi=-$ CiRT. Dónde: $\Psi \pi=$ potencial osmótico, $-\mathrm{C}=$ concentración de la solución expresada como molaridad (moles de soluto por $\mathrm{kg}$ de agua), $i$ $=$ constante para la ionización del soluto (0.00545), $R=$ constante de los gases $\left(0.00831 \mathrm{~kg} \mathrm{MPa} \mathrm{mol}^{-1}\right.$ $\left.\mathrm{K}^{-1}\right), T=$ temperatura en grados Kelvin (298.15 $\left.{ }^{\circ} \mathrm{K}\right)$.

Se utilizaron cajas Petri de $90 \mathrm{~mm}$ de diámetro provistas de algodón y papel filtro. La unidad experimental fue la caja Petri con 50 semillas, se utilizaron cuatro repeticiones por genotipo y nivel osmótico. Cada repetición fue 
humectada con $25 \mathrm{ml}$ iniciales de la solución correspondiente y se suministró riegos por aspersión de $2 \mathrm{ml}$ cada tercer día. Las cajas Petri se colocaron en una cámara de crecimiento (Precision Scientific, modelo $6 \mathrm{M}$ ) a temperatura de $28 \pm 2$ ${ }^{\circ} \mathrm{C}$. Se consideró como semilla germinada aquella que alcanzó $0.5 \mathrm{~cm}$ de plúmula o radícula. Las variables evaluadas fueron porcentaje germinación (GE), índice de velocidad de germinación (Vge), longitud de radícula (LR) y longitud plúmula (LP). Para obtener LR y LP se dejaron crecer las plántulas siete días después de las primeras germinadas, para lo cual se consideraron tres plantas por caja petri. La Vge se calculó mediante la ecuación propuesta por Maguire (1962): $V g e=\sum \frac{\left(n_{i}\right)}{t}$. Dónde: $V g e=$ velocidad de germinación, $n=$ número de semillas germinadas por día, $t=$ día de la germinación.

El experimento se realizó bajo un diseño completamente al azar y el modelo estadístico para el análisis de los datos correspondió a un arreglo de dos factores (genotipo y nivel osmótico) de la siguiente manera: $Y_{i j}=\mu+V_{i}+N_{j}+V_{*} N_{j}+\varepsilon_{i j k}$. Dónde: $Y_{i j}=$ variable de respuesta, $\mu=$ media general, $V_{i}=$ efecto fijo del $i$-ésimo genotipo, $N_{j}=$ efecto fijo aleatorio del $j$-ésimo nivel osmótico, $V_{i} * N_{j}=$ efecto fijo aleatorio de la interacción entre el $i$-ésimo genotipo y el $j$-ésimo nivel osmótico, $\varepsilon_{i j k}=$ error experimental.

Los datos se analizaron con un análisis de varianza (ANDEVA), la comparación de medias se realizó por medio de la prueba de Tukey. Los datos de germinación se trasformaron por medio del arco-seno (seno-1). Sin embargo, para una mayor aproximación de las estimas a la realidad, las figuras se realizaron con los datos originales. El análisis de los datos se realizó con el procedimiento GLM de SAS.

\section{RESULTADOS Y DISCUSIÓN}

La Figura 1 muestra el porcentaje de GE y Vge de los genotipos evaluados. Para GE se tuvieron diferencias $(p<0.001)$ en el comportamiento de los genotipos a través de los niveles osmóticos. El efecto de interacción en las variables genotipo- presión osmótica $(\mathrm{p}<0.05)$ estuvo relacionado con la germinación $\left(R^{2}=0.97\right)$. Para el nivel osmótico $0.0 \mathrm{MPa}$ no se observó diferencia $(p>0.05)$ entre E-592, Va, Ni, Re y K-13. No obstante, estos presentaron mayor GE $(p<0.05)$ que E-689 y K-07 (Figura 1a). El genotipo K-07 presentó menor GE $(p<0.05)$ comparado con el resto. En el nivel -0.7 MPa, los genotipos E-592, Va y K-13 no presentaron diferencias en GE $(p>0.05)$ y tuvieron valores de germinación mayores $(p<0.05)$ que E-689, Ni, Re y K-07. Los genotipos $\mathrm{Va}$ y E592 presentaron los valores más altos $(p<0.05)$ a -1.4 MPa con respecto a los demás y mantuvieron su germinación sin presentar diferencias ( $p$ $>0.05$ ) entre los niveles $0.0,-0.7$ y $-1.4 \mathrm{MPa}$. En este nivel, el valor más bajo $(\mathrm{p}<0.05)$ lo presentó K-07 con $49 \%$. Este resultado es similar a lo reportado por Hardegree y Emmerich (1994) quienes evaluaron la respuesta de germinación en ecotipos de pasto banderita recolectados en Tucson, Arizona. Se tuvo un $58 \%$ de germinación a $-1.5 \mathrm{MPa}$ con polietilenglicol, Valor inferior al presentado por el resto de los genotipos evaluados. Esto podría indicar que la semilla de estos genotipos tienen mayor potencial de turgencia. En otro estudio, Biligetu et al. (2011) al utilizar el mismo agente osmótico obtuvieron $48 \%$ de germinación a -1.2 MPa en semilla de la misma especie. De igual manera este resultado es inferior al presentado por la mayor parte de los genotipos evaluados. Lo anterior, posiblemente se debe a que los materiales evaluados en este estudio fueron recolectados en diferentes localidades de las praderas canadienses, donde las condiciones de sequía no son tan extremas como las del lugar de originen de los genotipos evaluados. Los genotipos $\mathrm{E}-592$ y Va presentaron la mayor GE $(\mathrm{p}<0.05)$ aún en -2.1 MPa, comparado con el resto de los genotipos. El genotipo Ni solo presentó potencial de germinación hasta -1.4 $\mathrm{MPa}$, ya que al ser sometido a -2.1 MPa no germinó. Lo cual podría indicar que no resiste altos niveles de estrés hídrico.

En especies utilizadas en programas de revegetación como pasto gigante (Leptochloa dubia), la máxima germinación alcanzada a un nivel osmótico de -2.1 MPa fue de 17 \% (Espinoza y Ku- 

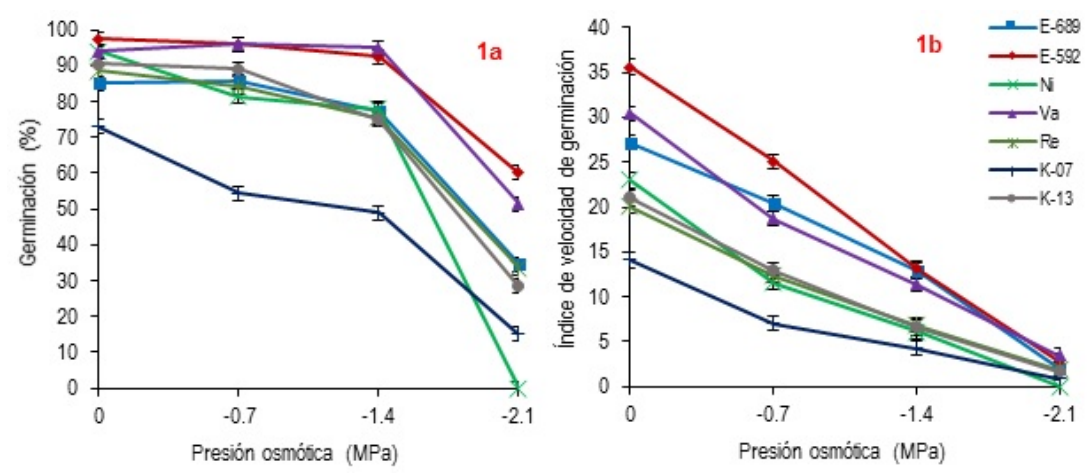

Figura 1. Porcentaje (a) y velocidad de germinación (b) de siete genotipos de pasto Banderita (Bouteloua curtipendula), bajo diferentes niveles de presión osmótica.

ruvadi 1985). Mientras que en zacate buffel (Pennisetum ciliare) la germinación obtenida fue del 50 $\%$ a - 2.0 MPa (Ruiz y Torenti 2012). En pasto africano (Eragrostis Lehmanniana) tuvo un $30 \%$ de germinación a $-1.2 \mathrm{MPa}$; sin embargo, a -1.5 MPa esta especie pierde la capacidad para germinar (Hardegree y Emmerich 1994). Por otro lado, especies como Agropyron elongatum y A. desertorum han alcanzado hasta un 45 y $56 \%$ de germinación, a -1.2 MPa (Zandi y Azarnivand 2012). Por lo anterior, la GE de los genotipos E-592 (60\%) y Va $(51.5 \%)$ a $-2.1 \mathrm{MPa}$ indica que pueden tener potencial para su inclusión en programas de restauración de pastizales en zonas áridas.

En la Vge (Figura 1b) se observó efecto ( $p<$ $0.001)$ por interacción genotipo-presión osmótica. El genotipo E-592 presentó la mayor $(p<0.05)$ Vge en los niveles 0.0 y $-0.7 \mathrm{MPa}$. Al respecto $\mathrm{Ab}$ bott y Roundy (2003) indican que especies como el pasto africano tiene mayor habilidad para controlar su germinación durante mayor tiempo y asegurar el establecimiento en zonas de precipitación errática. Contrario a lo anterior, especies con alto índice de germinación están sujetas a la muerte de plántulas después de un evento de lluvia. En este sentido, el pasto banderita presenta altas tasa de mortalidad durante sus primeras etapas de desarrollo en condiciones de sequía, debido a su alta velocidad de germinación (Esqueda et al. 2005). Por lo anterior, los resultados de Vge del genotipo E-592 a 0.0 y -0.7 MPa (35.6 y 25.1) podrían ser una desven- taja para su establecimiento. Además, en los niveles -1.4 y -2.1 MPa el genotipo E-592 y Va presentaron mayor $(p>0.05)$ Vge, mientras que K-13 y Re mostraron una Vge media. El genotipo K-07 mostró la menor $(p<0.05)$ Vge en todos los niveles osmóticos con excepción del nivel -2.1 MPa, donde $\mathrm{Ni}$ al no germinar no presentó valores de Vge. La Figura 2 muestra el comportamiento de la GE y Vge de los genotipos evaluados; el mayor porcentaje de germinación se presentó antes de los primeros seis días de evaluación, donde los genotipos Re, E-689 y K-13 presentaron una germinación más dispersa. El comportamiento de especies con alta velocidad de germinación podría ser una ventaja al competir con malezas y disponibilidad de humedad durante la etapa de establecimiento. Sin embargo, una desventaja en zonas con precipitación errática sería el agotamiento del banco de semillas.

En LP se observó diferencia $(p<0.05)$ en el comportamiento de los genotipos a través de los niveles osmóticos (Figura 3a). El efecto de la interacción genotipo-presión osmótica fue significativo en los genotipos $\left(p<0.05 ; R^{2}=0.78\right)$. Los genotipos $\mathrm{Va}, \mathrm{E}-592$ y E-689 presentaron la mayor $(p<0.05)$ LP hasta el nivel de $1.4 \mathrm{MPa}$, sin presentar diferencias $(p>0.05)$ entre ellos. Tampoco se encontró diferencia $(p>0.05)$ en el comportamiento de $\mathrm{Re}, \mathrm{Ni}, \mathrm{K}-13$ y K-07 en la mayoría de los niveles osmóticos. En LP no se presentaron diferencias $(p>0.05)$ entre genotipos a -2.1 MPa. La longitud de la plúmula que presentaron $\mathrm{Va}$ (34.8 


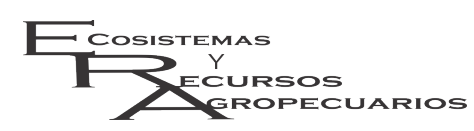

K-6113

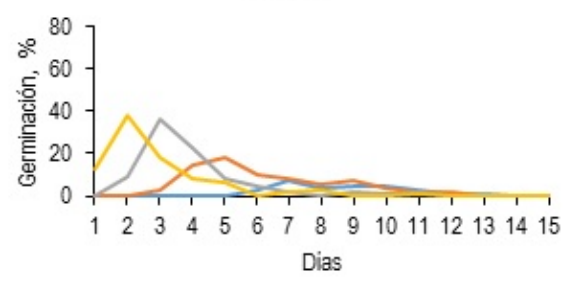

Niner

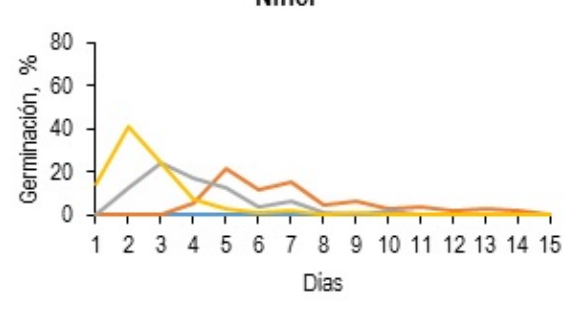

689
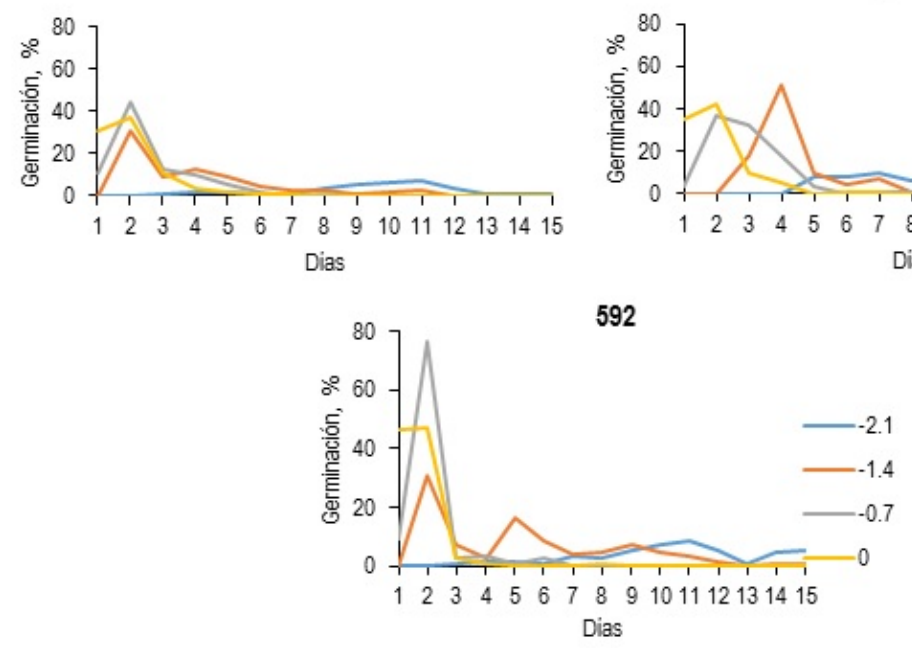

K-6107
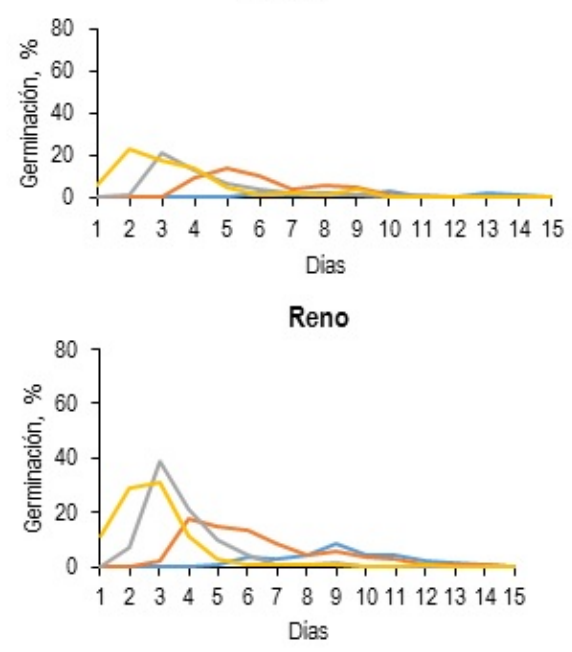

Vaughn

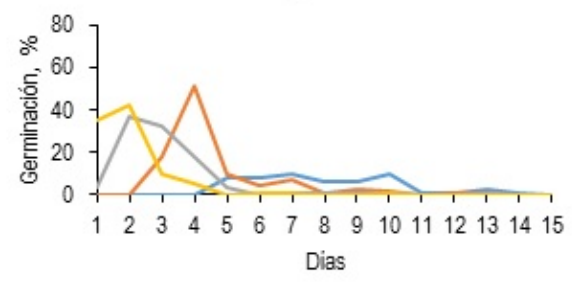

Figura 2. Comportamiento diario de germinación de siete genotipos de pasto Banderita (Bouteloua curtipendula), bajo diferentes niveles de presión osmótica $(0.0,-0.7,-1.4$ y -2.1 MPa).

$\mathrm{mm})$, E-592 (39.1 mm) y E-689 (39.6 mm) a 0 MPa fue similar a lo reportado por González et al. (2011), quienes obtuvieron LP de $35 \mathrm{~mm}$ en zacate banderita al noveno día de la emergencia. Por otro lado, Carren et al. (1987) reportaron LP de $58 \mathrm{~mm}$ en pasto navajita, mientras que en otras especies introducidas como zacate buffel (Pennisetum ciliare) y garrapata (Eragrostis superba) se han registrado longitudes de 60 y $120 \mathrm{~mm}$, respectivamente, durante la etapa de plántula (Rasool et al. 2012).

Un aspecto importante durante el estableci- miento de una planta es el desarrollo de la plúmula después de la germinación, ya que durante este periodo la planta es susceptible a efectos de déficit hídrico y daños ocasionados por depredadores (Raven et al. 1991). Además, una plúmula más desarrollada al término de esta etapa podría representar una mayor capacidad para captar recursos fotosintéticos cuando los nutrientes de la semilla terminan y la planta tiene que ser autosuficiente (Rodés y Collazo 2006). De acuerdo a lo anterior, los valores obtenidos en longitud de plúmula de los genotipos 

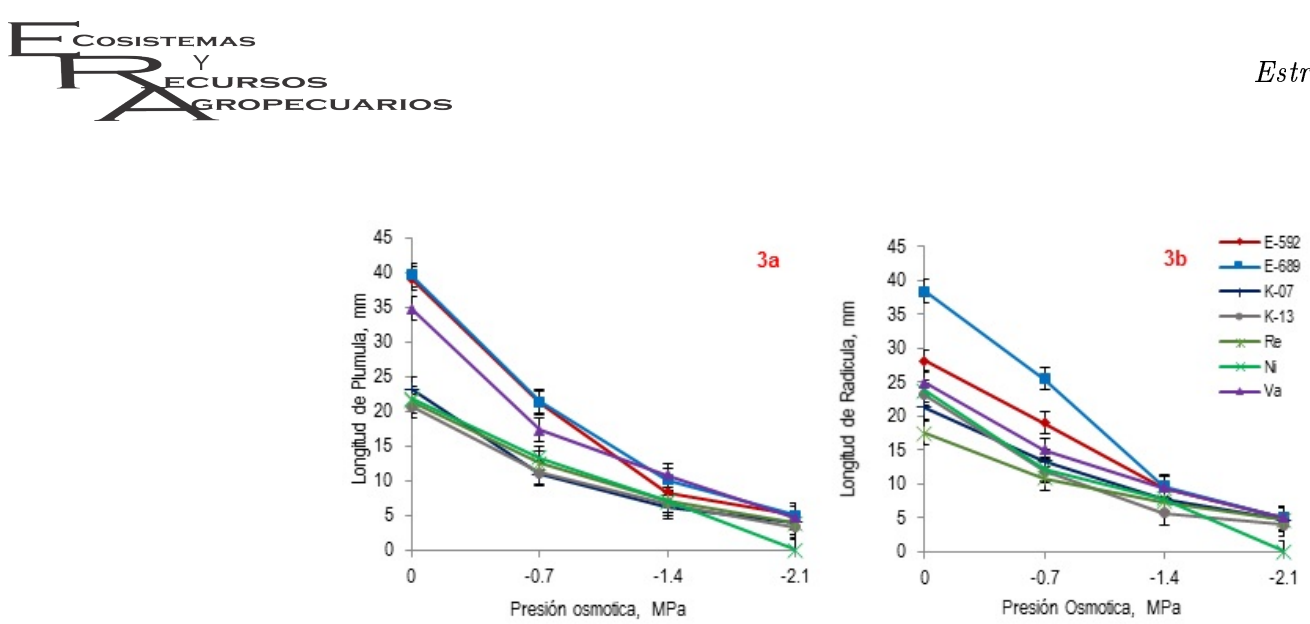

Figura 3. Longitud de plúmula (a) y radícula (b) de siete genotipos de pasto banderita (Bouteloua curtipendula), bajo diferentes niveles de presión osmótica en siete días de germinación.

Va, E-592 y E-689 podrían tener mayor capacidad para establecerse en condiciones de sequía.

En el comportamiento de LR se observó diferencias $\left(p<0.001 ; R^{2}=0.78\right.$ ) entre genotipos por nivel osmótico (Figura $3 \mathrm{~b}$ ). En el nivel 0.0 $\mathrm{MPa}$, el genotipo E-689 presentó la mayor $(\mathrm{p}<$ $0.05) \mathrm{LR}$, mientras que en los otros genotipos no se encontraron diferencias $(p>0.05)$. Al respecto González et al. (2011) reportaron una LR de 22 $\mathrm{mm}$ al noveno día de iniciada la germinación. Este resultado es menor al que presento el genotipo E$689(38.4 \mathrm{~mm})$, lo cual podría indicar un potencial en este genotipo para su establecimiento. Por otro lado, estudios realizados por Jurado et al. (2011) reportan una LR de $55 \mathrm{~mm}$ a los $30 \mathrm{~d}$ post emergencia en esta misma especie. En especies introducidas como pasto buffel (Pennisetum ciliare) y garrapata (Eragrostis superba) durante la etapa de germinación se han reportado longitudes de radícula de 9 y $8 \mathrm{~mm}$, respectivamente (Rasool et al. 2012). Los genotipos que mostraron mayor $(p<0.05)$ LR a -0.7 MPa fueron E-689 y E-592. Sin embargo, a -1.4 MPa, el genotipo K-07 fue el único que mostró diferencias $(p<0.05)$ con la menor LR, mientras que a -2.1 MPa no se presentaron diferencias ( $p>$ $0.05)$ entre genotipos.

La LR es una característica importante para la supervivencia de pastos en programas de rehabilitación, donde el establecimiento es dificil y es una de las principales razones por la que este tipo de programas fracasan. Además, es importante considerar que en las zonas áridas se presenta un rápido desecamiento de la superficie del suelo, lo cual representa una desventaja para la supervivencia de plántulas. También, una raíz con mayor longitud podría representar una ventaja en lugares donde los eventos de lluvia son escasos, ya que esto permite aprovechar mejor el agua a una mayor profundidad (Sala y Laurenroth 1982, Busso y Bolletta 2007, Ervin et al. 2009). Además, un mayor desarrollo tanto de radícula como de plúmula puede facilitar el establecimiento de plantas por una mejor asimilación de nutrientes (Raven et al. 1991). Considerando los aspectos anteriores, los genotipos E592, E-689 y Vauhgn de este estudio presentaron la mayor longitud de radícula y plúmula.

Los genotipos E-592 y Vauhgn fueron los más resistentes al estrés hídrico; no obstante, presentaron la mayor velocidad de geminación. Estos resultados pueden considerarse para su inclusión en programas de resiembras de pastizales. De acuerdo a lo anterior, se recomienda combinar los genotipos E-592 y Vauhgn con El Reno o Kansas 6113 (con menor velocidad de germinación), lo cual podría incrementar la probabilidad de éxito en programas de rehabilitación de agostaderos en zonas áridas. Los genotipos E-592 y E-689 presentaron mayor longitud de radícula y plúmula en los niveles de 0.0 y -0.7 Mpa, lo que pudieran representar una ventaja para competir por nutrientes y humedad, facilitar su establecimiento. 


\section{LITERATURA CITADA}

Aasamaa K, Sõeber A (2011) Stomatal sensitivities to changes in leaf water potential, air humidity, $\mathrm{CO}^{2}$ concentration and light intensity, and the effect of abscisic acid on the sensitivities in six temperate deciduous tree species. Environmental and Experimental Botany 71: 72-78.

Abbott LB, Roundy BR (2003) Available water influences field germination and recruitment of seeded grasses. Journal of Range Management 56: 56-64.

Aparecida DME, Zambillo de PS (2003) Germination of Senna occidentales link: Seed at different osmotic potential levels. Brazilian Archives of Biology and Technology 46: 38-43.

Beltrán LS, Garcia DCA, Hernández AJA, Loredo OC, Urrutia MJ, González ELA, et al. (2010) "Navajita Cecilia" Bouteloua gracilis H.B.K (Lag.). Nueva variedad de pasto para zonas áridas y semiáridas. Revista Mexicana de Ciencias Pecuarias 1: 127:130.

Biligetu B, Schellenberg MP, McLeod JG (2011) The effect of temperature and water potential on seed germination of poly-cross side-oats grama (Bouteloua curtipendula (Michx.) Torr.) population of Canadian prairie. Seed Science and Technology 39: 74-81.

Busso CA, Bolletta AI (2007) Perennial grasses of different successional stages under various soil water inputs: Do they differ in root length density? Interciencia 32: 206-212.

Carren, CJ, Wilson AM, Cuany RL, Thor GL (1987) Caryopsis weight and planting depth of blue grama I. Morphology, emergence, and seedling growth. Journal of Range Management 40: 207-211.

Ervin EH, La-Branche A, Zhang X (2009) Kentucky bluegrass and creeping bentgrass responses to foliar application of glycinebetaine at three replacement levels. International Turfgrass Society 11: 755-763.

Espinoza ZR, Kuruvadi S (1985) Clasificación de colecciones de zacate gigante (Leptochloa dubia HBK, Ness) por su grado de resistencia a sequía en manitol. Revista Agraria Científica 1: 142-152.

Esqueda CMH, Melgoza CA, Sosa CM, Carrillo RR, Jiménez CJ (2005) Emergencia y sobrevivencia de gramíneas con diferentes secuencias de humedad/sequía en tres tipos desuelo. Técnica Pecuaria en México 43: 101-115.

González RSL, Quero CAR, Franco MO, Ramírez AC, Ortega EHM, Trejo LC (2011) Efecto de la salinidad y la temperatura sobre el crecimiento del pasto banderita [Bouteluoa curtipendula (Michx.) Torr.]. Ciencia Ergo Sum 18: 59-69.

Hardegree SP, Emmerich WE (1994) Seed germination response to polyethylene glycol solution depth. Seed Science and Technology 22: 1-7.

Jurado GP, Domínguez CH, Melgoza CA, Morales NC (2011) Emergencia y crecimiento del zacate banderilla con biosólidos en condiciones de sequía. Tecnociencia Chihuahua 6: 57-67.

Lloyd-Reilley J, Smith F (2012) Plant guide for South Texas Germplasm sideoats grama. USDA-Natural Resources Conservation Service. http://www.nrcs.usda.gov/Internet/FSE_PLANTMATERIALS/publica tions/stpmcpg11359.pdf. Fecha de consulta 5 de Abril de 2015.

Maguire JD (1962) Speed of germination-aid in selection and evaluation for seedling emergence and vigor. Crop Science 2: 176-177.

Méndez NJ, Ybarra PF, Merazo PJ (2010) Germinación y desarrollo de plántulas de tres híbridos de maíz bajo soluciones osmóticas. VI. Comparación entre cinco soluciones osmóticas. Revista Tecnológica ESPOL 23: 55-60. 
Morales NCR, Quero CA, Melgoza CA, Martínez SM, Jurado GP (2009) Diversidad forrajera del pasto banderita [Bouteloua curtipendula (Michx.) Torr.], en poblaciones de zonas áridas y semiáridas de México. Técnica Pecuaria en México 47: 231-244.

Morales-Nieto C, Quero-Carrillo A, Le-Blanc O, Hernández-Garay A, Pérez-Pérez J, González-Muñoz S (2006) Caracterización de la diversidad del pasto nativo Bouteloua curtipendula Michx. Torr. mediante marcadores de AFLP. Agrociencia 40: 711-720.

Rassol F, Ishaque M, Khan ZH, Hussain Z, Khalid KM, Muhammad W (2012) Growth comparison of some indigenous and exotic grasses at germination and seedling stage. World Applied. Sciences Journal 19: 1437-1442.

Raven PH, Evert RF, Eichhorn SE (1991) Biología de las plantas, volumen 2. 1a ed. Editorial Reverté. S. A. Barcelona, España. 541p.

Rodés GR, Collazo OM (2006) Manual de prácticas de fotosíntesis. 1a ed. Editorial las prensas de ciencias, UNAM. México. $7 \mathrm{p}$.

Ruiz M, Torenti O (2012) Germinación de cuatro pastos bajo condiciones de estrés salino. Phyton Revista Internacional de Botánica Experimental 81: 169-176.

Sala OE, Lauenroth WK (1982) Small rainfall events: An ecological role in semiarid regions. Oecologia 53: 301-304.

Silmara FC, Juliano PSC (2004) Processo germinativo de sementes de paneira sobre estrés hídrico e salino. Pesquisa Agropecuária Brasileira 39: 24-31.

Springer TL (2005) Germination and early seedling growth of chaffy-seeded grasses at native water potentials. Crop Science 45: 2075-2080.

Townsend AP, Ortega-Huerta AM, Bartley J, Sánchez-Cordero V, Soberón J, Buddemeier RH, Stockwell DRB (2002) Future projections for Mexican faunas under global climate change scenarios. Nature 416: 626-629.

Trachsel S, Stamp P, Hund A (2010) Effect of high temperatures, drought and aluminum toxicity on root growth of tropical maize (Zea mays L.) seedlings. Maydica 55: 249-260.

Zandi EE, Azarnivand H (2012) Effect of water stress on seed germination of Agropyron elongatum, Agropyron desertourm \& Secale montanu. Desert 7: 249-253. 